\title{
Superconducting transition temperatures and coherence length in non-s -wave pairing materials correlated with spin-fluctuation mediated interaction
}

\author{
G. G. N. Angilella, ${ }^{1}$ N. H. March, ${ }^{2,3}$ and R. Pucci ${ }^{1}$ \\ ${ }^{1}$ Dipartimento di Fisica e Astronomia, Università di Catania, and Istituto Nazionale per la Fisica della Materia, \\ UdR di Catania, Corso Italia, 57, I-95129 Catania, Italy \\ ${ }^{2}$ Oxford University, Oxford, England \\ ${ }^{3}$ Department of Physics, University of Antwerp (RUCA), Antwerp, Belgium
}

(Received 18 July 2001; published 13 February 2002)

\begin{abstract}
Following earlier work on electron or hole liquids flowing through assemblies with magnetic fluctuations, we have recently exposed a marked correlation of the superconducting temperature $T_{c}$, for non- $s$-wave pairing materials, with coherence length $\xi$ and effective mass $m^{*}$. The very recent study of Abanov et al. [Europhys. Lett. 54, 488 (2001)] and the prior investigation of Monthoux and Lonzarich [Phys. Rev. B 59, 14598 (1999)] have each focused on the concept of a spin-fluctuation temperature $T_{\text {sf }}$, which again is intimately related to $T_{c}$. For the $d$-wave pairing via antiferromagnetic spin fluctuations in the cuprates, these studies are brought into close contact with our own work, and the result is that $k_{\mathrm{B}} T_{\mathrm{sf}} \sim \hbar^{2} / m^{*} \xi^{2}$. This demonstrates that $\xi$ is also determined by such antiferromagnetic spin-fluctuation mediated pair interaction. The coherence length in units of the lattice spacing is then essentially given in the cuprates as the square root of the ratio of two characteristic energies, namely, the kinetic energy of localization of a charge carrier of mass $m^{*}$ in a specified magnetic correlation length to the hopping energy. The quasi-two-dimensional ruthenate $\mathrm{Sr}_{2} \mathrm{RuO}_{4}$, with $T_{c} \sim 1.3 \mathrm{~K}$, has $p$-wave spin-triplet pairing and so is also briefly discussed here.
\end{abstract}

DOI: 10.1103/PhysRevB.65.092509

PACS number(s): 74.72.-h, 74.70.Pq, 74.70.Tx

\section{INTRODUCTION}

In early work, Egorov and March $^{1}$ discussed electron or hole liquids flowing through assemblies with antiferromagnetic spin fluctuations, and proposed a correlation between the in-plane electrical resistivity $\rho_{a b}$ and the nuclear-spinlattice relaxation time $T_{1}$ of the form

$$
\rho_{a b} T_{1} \propto T .
$$

This relationship has been tested on the underdoped high- $T_{c}$ cuprate $\mathrm{YBa}_{2} \mathrm{Cu}_{4} \mathrm{O}_{8}$ with $T_{1}$ extracted from ${ }^{63} \mathrm{Cu}$ nuclear magnetic resonance (NMR) data, and is appropriate somewhat above the superconducting transition temperature (see Fig. A.7.5.1 on p. 355 of Ref. 2 and Ref. 3). The correlation written in Eq. (1) between the observables $\rho_{a b}$ and $T_{1}$ arose $^{1}$ by eliminating the magnetic susceptibility $\chi(\mathbf{Q})$, at the antiferromagnetic wave vector $\mathbf{Q}$ [equal to $(\pi / a, \pi / a)$ in the treatment below, $a$ being the lattice spacing], with $\rho_{a b}$ and $\left(T T_{1}\right)^{-1}$ both proportional to $\chi(\mathbf{Q})$, as shown by Kohno and Yamada. ${ }^{4}$

More recently, the present authors ${ }^{5}$ have demonstrated that $T_{c}$ for non- $s$-wave pairing superconductors, and, in particular, for heavy fermion materials and high- $T_{c}$ cuprates, correlated with coherence length and effective mass. The very recent and apparently quite different studies of Abanov et al., ${ }^{6}$ and prior to that, of Monthoux and Lonzarich, ${ }^{7}$ are here brought into close contact with our earlier work. ${ }^{5}$

The outline of this paper is then as follows. In Sec. II, we briefly summarize the essential input in the treatments of spin-fluctuation mediated pairing in Refs. 6 and 7. Anticipating that, we stress at the outset that the common feature in Refs. 6 and 7 is a characteristic thermal energy $k_{\mathrm{B}} T_{\text {sf }}$ associated with a spin-fluctuation temperature $T_{\mathrm{sf}}$. Section III connects the work on $T_{\text {sf }}$ in Refs. 6 and 7 with our own studies on coherence length. A summary is then given in Sec. IV, with some possible directions for future work.

\section{SPIN-FLUCTUATION TEMPERATURE RELATED TO $T_{c}$ IN $p$ - AND $d$-WAVE SUPERCONDUCTIVITY IN QUASI-2D METALS}

Essential input into both Refs. 6 and 7 is a form of the retarded generalized magnetic susceptibility $\chi(\mathbf{q}, \omega)$. Quite specifically, in Ref. 7 the phenomenological form

$$
\chi(\mathbf{q}, \omega)=\frac{\chi_{0} \kappa_{0}^{2}}{\kappa^{2}+\hat{q}^{2}-i[\omega / \eta(\hat{q})]}
$$

is assumed. Here, $\kappa^{-1}$ and $\kappa_{0}^{-1}$ are correlation lengths in units of the lattice constant $a$, with and without strong magnetic correlations, respectively.

Monthoux and Lonzarich ${ }^{7}$ then adopt a tight-binding form for the quasiparticle dispersion relation, and subsequently define the quantities $\hat{q}_{ \pm}^{2}$ by

$$
\hat{q}_{ \pm}^{2}=4 \pm 2\left[\cos \left(q_{x} a\right)+\cos \left(q_{y} a\right)\right] .
$$

In the case of ferromagnetic correlations, typified by the ruthenate $\mathrm{Sr}_{2} \mathrm{RuO}_{4}$ with a low $T_{c} \sim 1.3 \mathrm{~K},{ }^{8}$ the parameters $\hat{q}^{2}$ and $\eta(\hat{q})$ entering $\chi(\mathbf{q}, \omega)$ in Eq. (2) are defined as

$$
\hat{q}^{2}=\hat{q}_{-}^{2}
$$

and

$$
\eta(\hat{q})=T_{\text {sf }} \hat{q}_{-},
$$


where $T_{\mathrm{sf}}$ is the spin-fluctuation temperature already referred to in Sec. I.

Here, we should caution that the use of a single-band dispersion relation [Eq. (3) in Ref. 7], while appropriate for most high- $T_{c}$ cuprates, is clearly a rather crude approximation for $\mathrm{Sr}_{2} \mathrm{RuO}_{4}$. Indeed, this compound is known to be characterized by a threefold band, arising from the hybridization of the ruthenium $4 d_{i j}$ atomic orbitals $(i j$ $=x y, x z, y z)$ in the $t_{2 g}$ subshell. As a consequence, the Fermi surface is composed of a set of three disconnected barrel-like sheets, almost dispersionless in the direction orthogonal to the $\mathrm{SrO}_{2}$ layers. ${ }^{9}$ This led to the proposal of an orbitaldependent form of superconductivity ${ }_{10}^{10}$ where the role of the multiband nature of $\mathrm{Sr}_{2} \mathrm{RuO}_{4}$ in stabilizing a $p$-wave triplet order parameter against a $d$-wave singlet alternative has been also emphasized. ${ }^{11}$ However, we believe that the main conclusions of Monthoux and Lonzarich ${ }^{7}$ would be unaffected by multiband effects, at least qualitatively. Moreover, the restriction to the main band of $\mathrm{Sr}_{2} \mathrm{RuO}_{4}$ [Eq. (3) in Ref. 7] helps treating the quasi-two-dimensional (2D) character of the single-particle dispersion relation of this layered perovskite on the same footing as for the cuprates. ${ }^{12}$

Monthoux and Lonzarich ${ }^{7}$ also investigated antiferromagnetic correlations as in the $d$-wave paired cuprates, in which case the above parameters have the form

$$
\hat{q}^{2}=\hat{q}_{+}^{2}
$$

and

$$
\eta(\hat{q})=T_{\text {sf }} \hat{q}_{-} .
$$

The final input we need to refer to here is a coupling parameter $g^{2}$ in the quasiparticle self-energy $\Sigma(\mathbf{q}, \omega)$, involving, of course [see Ref. 7, Eqs. (11)-(13)], summations over wave vectors and Matsubara frequencies of $\chi(\mathbf{q}, \omega)$.

The mean-field Eliashberg equations for nearly ferromagnetic and nearly antiferromagnetic metals with a single $2 \mathrm{D}$ Fermi surface were then solved numerically in Ref. 7, to obtain the ratio of critical temperature $T_{c}$ to spin-fluctuation temperature $T_{\text {sf }}$ essentially as a function of coupling strength $g^{2}$ for different values of the inverse correlation length $\kappa$. This was done both for $p$-wave triplet and $d$-wave singlet pairing.

The major predictions of Refs. 6 and 7 were in accord that at strong coupling, $T_{c} / T_{\text {sf }}$ exhibits saturation. For a physically reasonable range of values of $\kappa^{-1}$, the quantitative results of Ref. 7 were (i) for $p$-wave triplet pairing, $T_{c} / T_{\text {sf }}$ saturates at a value of $1 / 30$, and (ii) for $d$-wave singlet pairing, $T_{c} / T_{\text {sf }}$ has a saturation value of $1 / 2$. This then is the point at which to make contact between these findings of Refs. 6 and 7 and our own study.

\section{SPIN-FLUCTUATION TEMPERATURE $\boldsymbol{T}_{\mathrm{sf}}$ AND CORRELATION LENGTH $\xi$ IN NON-s-WAVE PAIRING SUPERCONDUCTORS: ESPECIALLY HIGH- $T_{c}$ CUPRATES}

In Ref. 5 we exposed a relationship for both heavy fermion materials and for high- $T_{c}$ cuprates, between the ther- mal energy $k_{\mathrm{B}} T_{c}$ and another characteristic energy $\epsilon_{c}$, say, for such non-s-wave superconductors, where $\epsilon_{c}$ was defined by

$$
\epsilon_{c}=\frac{\hbar^{2}}{m^{*} \xi^{2}},
$$

$\xi$ being the coherence length and $m^{*}$ the effective mass. We noted $^{5}$ that Uemura et al. ${ }^{13}$ had already clearly recognized that $m^{*}$ should enter inversely in determining the scale of $k_{\mathrm{B}} T_{c}$.

Since Monthoux and Lonzarich ${ }^{7}$ made their most extensive numerical investigations for the $p$-wave triplet pairing, let us take first the ruthenate $\mathrm{Sr}_{2} \mathrm{RuO}_{4}$, discussed at some length in Ref. 7. From Fig. 2(a) of Ref. 7, for example, provided $\kappa^{2}$ is in the (physically reasonable) range from 0.25 to 1.0 and their quantity $g^{2} \chi_{0} / t$, with $t$ the hopping energy, is in the physical range 10-20, then $T_{c} / T_{\mathrm{sf}}$ is in the range $0.02-0.03$, which yields $T_{\mathrm{sf}} \simeq 50 \mathrm{~K}$ for $\mathrm{Sr}_{2} \mathrm{RuO}_{4}$, compared with a "saturation" value of $40 \mathrm{~K}$ for this material with $T_{c}$ $\sim 1.3 \mathrm{~K}$. Abanov et al. ${ }^{6}$ refer to values of $T_{\mathrm{sf}} \sim 100 \mathrm{~K}$, so there is semiquantitative accord.

Turning to the high- $T_{c}$ cuprates, the present authors ${ }^{5}$ have pointed out that in marked contrast to the heavy fermion materials they also considered, $k_{\mathrm{B}} T_{c} \sim \hbar^{2} / m^{*} \xi^{2}$ and from the saturation limit (ii) in Sec. II, taken again from Ref. 7,

$$
k_{\mathrm{B}} T_{c} \sim \frac{1}{2} k_{\mathrm{B}} T_{\mathrm{sf}}
$$

for the $d$-wave pairing high- $T_{c}$ cuprates. Thus, one has as a consequence the order of magnitude result

$$
k_{\mathrm{B}} T_{\mathrm{sf}} \sim \frac{2 \hbar^{2}}{m^{*} \xi^{2}},
$$

and the coherence length $\xi$ of the high- $T_{c}$ cuprates is plainly determined by the antiferromagnetic spin-fluctuation mediated pairing, via a temperature $T_{\mathrm{sf}} \sim 2 T_{c}$.

\section{Physical interpretation of the coherence length $\xi$ resulting from $d$-wave singlet pairing spin-fluctuation interaction in the high- $T_{c}$ cuprates}

We return to our earlier result ${ }^{5}$ obtained from experimental data for the high- $T_{c}$ cuprates that $k_{\mathrm{B}} T_{c} \sim \epsilon_{c}$, with $\epsilon_{c}$ given by Eq. (8). We now add to this empirical correlation a further experimental consequence used by Monthoux and Lonzarich, ${ }^{7}$ namely, that the product of the thermal energy $k_{\mathrm{B}} T_{\text {sf }}$ associated with the spin-fluctuation temperature ${ }^{6,7} T_{\mathrm{sf}}$ with $\kappa_{0}^{2}$, the inverse magnetic correlation length squared without strong magnetic correlations, is constant, i.e.,

$$
k_{\mathrm{B}} T_{\mathrm{sf}} \kappa_{0}^{2}=\text { const } .
$$

Adopting the value in their Table V, the constant value turns out to be $\sim 8 t$. 
Returning to the strong coupling limit to gain further insight into the factors determining the coherence length $\xi$, we have

$$
k_{\mathrm{B}} T_{c} \sim \frac{1}{2} k_{\mathrm{B}} T_{\mathrm{sf}} \sim \frac{4 t}{\kappa_{0}^{2}} .
$$

Putting $\kappa_{0}^{2}=a^{2} / l_{m 0}^{2}$, where $l_{m 0}$ is the (antiferro-) magnetic correlation length in the high- $T_{c}$ cuprates, we find almost immediately

$$
\xi \sim \frac{a}{2}\left(\frac{\hbar^{2}}{m^{*} l_{m 0}^{2}}\right)^{1 / 2} \frac{1}{t^{1 / 2}}
$$

Physically, Eq. (13) shows that, in units of the lattice spacing $a$, the magnitude of the coherence length is determined by the square root of the ratio of two further characteristic energies. The first of these is the kinetic energy of localization of a carrier of mass $m^{*}$ within the magnetic correlation length $l_{m 0}$ determined, however, in the absence of strong magnetic correlations. The second energy is $t$, the magnitude of the hopping energy.

We can anticipate, using the additional (generally weaker) variables $g^{2} \chi_{0} / t$ and $\kappa^{2}$ that, provided the range of $g^{2} \chi_{0} / t$ is limited to the physical region 10 to 20 (see Ref. 7) and $\kappa^{2}$ is likewise restricted to the range 0.5 to 1 , then Eq. (13) will be replaced, away from the strong coupling limit, by

$$
\xi \sim \frac{a}{2}\left(\frac{\hbar^{2}}{m^{*} l_{m 0}^{2}}\right)^{1 / 2} \frac{1}{t^{1 / 2}} F\left(g^{2} \chi_{0} / t ; \kappa^{2}\right),
$$

where $F$ is a slowly varying function of its arguments, $F$ becoming unity for sufficiently large values of the "coupling strength" $g^{2} \chi_{0} / t$, with $\kappa^{2}$ restricted to the range quoted above.

\section{SUMMARY AND DIRECTIONS FOR FUTURE WORK}

The achievement of the present paper is to bring the studies of Refs. 6 and 7, in which the superconducting transition temperature $T_{c}$ is connected to the spin-fluctuation temperature $T_{\text {sf }}$, into direct contact with our work relating $k_{\mathrm{B}} T_{c}$ to the characteristic energy $\hbar^{2} / m^{*} \xi^{25}$. For a $d$-wave singlet pairing mediated by antiferromagnetic spin fluctuations in the cuprates, the simple, order of magnitude relation Eq. (10) follows, showing that the coherence length $\xi$ is determined by the interaction mediated by spin fluctuations. This is expressed, more specifically, in the language of Ref. 7, in Eqs. (13) and (14).

However, the situation regarding the relation of $T_{\mathrm{sf}}$ in the low- $T_{c}$ ruthenate $\mathrm{Sr}_{2} \mathrm{RuO}_{4}$ to the coherence length $\xi$ is much less clear presently than in the high- $T_{c}$ cuprates. This may be because of a competition between nearly ferromagnetic behavior and antiferromagnetic spin fluctuations. ${ }^{11}$ Experiments on $\chi(\mathbf{q}, \omega)$ using both neutron scattering and NMR on this ruthenate would be valuable for further understanding of the origins of superconductivity, and especially the physics of the coherence length in this material. As for $T_{\text {sf }}$ it seems to lie in the range $40-50 \mathrm{~K}$. Having referred to heavy fermion materials in connection with Ref. 5, we thought it of interest

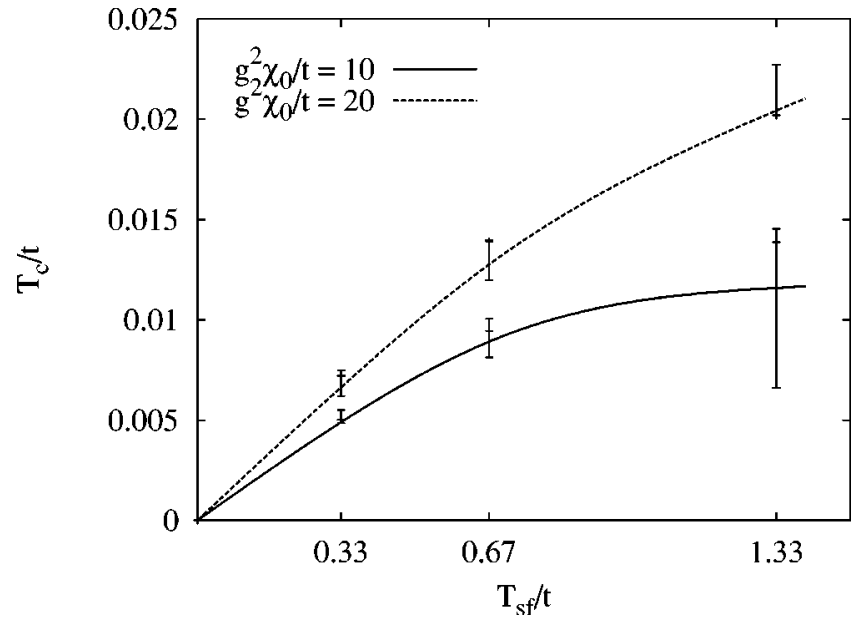

FIG. 1. Shows plot of $T_{c} / t$ vs $T_{\text {sf }} / t$ for $p$-wave spin-triplet pairing. This has been constructed by combining numerical data from Figs. 2-4 of Ref. 7, assuming for the parameters the physical range $10 \leqslant g^{2} \chi_{0} / t \leqslant 20$ and $0.25 \leqslant \kappa^{2} \leqslant 1$. Points corresponding to the same value of $T_{\mathrm{sf}} / t$ and of $g^{2} \chi_{0} / t$, but to different values of $\kappa^{2}$, have been arranged as vertical bars. The two curves shown are guides to the eye through the choices of "coupling strength" $g^{2} \chi_{0} / t$ of 10 and 20, and have been extrapolated through the origin.

to construct from the $p$-wave studies of Ref. 7, a plot of $T_{c} / t$ vs $T_{\text {sf }} / t$ (see Fig. 1), by combining data from their Figs. $2-4$. It is worth noting, though we expect the mechanisms generally to be different, that the shape of the present Fig. 1 parallels that of Fig. 1 of Ref. 5. However, such a comparison should be carefully considered, since some of the heavy fermion materials considered in Fig. 1 of Ref. 5 exhibit antiferromagnetic spin fluctuations, rather than ferromagnetic spin fluctuations, as studied by Monthoux and Lonzarich in connection with $p$-wave superconductors. ${ }^{7}$ The major exception is $\mathrm{UPd}_{2} \mathrm{Al}_{3}$, which is known to be characterized by rather strong, static antiferromagnetic correlations in the normal state (with a significant Néel temperature of $T_{\mathrm{N}}=14.3 \mathrm{~K}$ ). Such antiferromagnetic correlations even coexist with superconductivity below $T_{c} \sim 2 \mathrm{~K}$, at variance with other uranium-based heavy fermion compounds. ${ }^{14-16}$ We record, however, that this compound, with its relatively high $T_{c}$, helped Sato (Fig. 2 in Ref. 17) to "bridge the gap" between the low- $T_{c}$ heavy fermion compounds and the high- $T_{c}$ cuprates in establishing a correlation between $T_{c}$ and some magnetic ordering temperature (though related to a different kind of magnetic order in different compounds), much in the same spirit as in the present paper (compare also Fig. 2 of Ref. 5).

\section{ACKNOWLEDGMENTS}

One of us (N.H.M.) made his contribution to the present paper during a visit to the Physics Department, University of Catania, in the year 2001. Thanks are due to the Department for the stimulating environment and for much hospitality. G.G.N.A. thanks Dr. G. Sparn for stimulating discussions and correspondence, and acknowledges partial support from the EU through the FSE program. 
${ }^{1}$ S. Egorov and N. H. March, Phys. Chem. Liq. 28, 141 (1994).

${ }^{2}$ N. H. March, Electron Correlation in Atoms, Molecules and Condensed Phases (Plenum Press, New York, 1996), p. 354.

${ }^{3}$ G. G. N. Angilella, N. H. March, and R. Pucci, Phys. Chem. Liq. 38, 615 (2000).

${ }^{4}$ H. Kohno and K. Yamada, Prog. Theor. Phys. 85, 13 (1991).

${ }^{5}$ G. G. N. Angilella, N. H. March, and R. Pucci, Phys. Rev. B 62, 13919 (2000).

${ }^{6}$ Ar. Abanov, A. V. Chubukov, and A. M. Finkel'stein, Europhys. Lett. 54, 488 (2001).

${ }^{7}$ P. Monthoux and G. G. Lonzarich, Phys. Rev. B 59, 14598 (1999).

${ }^{8}$ Y. Maeno, H. Hashimoto, K. Yoshida, S. Nishizaki, T. Fujita, J. G. Bednorz, and F. Lichtenberg, Nature (London) 372, 532 (1994).

${ }^{9}$ A. P. Mackenzie, S. R. Julian, A. J. Diver, G. J. McMullan, M. P. Ray, G. G. Lonzarich, Y. Maeno, S. Nishizaki, and T. Fujita, Phys. Rev. Lett. 76, 3786 (1996).

${ }^{10}$ D. F. Agterberg, T. M. Rice, and M. Sigrist, Phys. Rev. Lett. 78, 3374 (1997).

${ }^{11}$ I. I. Mazin and D. J. Singh, Phys. Rev. Lett. 82, 4324 (1999).
${ }^{12}$ The role of reduced dimensionality in enhancing magnetically mediated superconductivity, as emphasized by Monthoux and Lonzarich (Ref. 7), has been recently confirmed experimentally in the layered heavy fermion compound $\mathrm{CeCoIn}_{5}$ (Ref. 18). There, pressure $P$ is employed to tune the anisotropy of this material. Qualitative agreement is found between Monthoux and Lonzarich's predictions and the experimental trend of $T_{c}$ $=T_{c}(P)$, with $P$ roughly identified with parameter $\kappa^{2}$ in Ref. 7.

${ }^{13}$ Y. J. Uemura et al., Phys. Rev. Lett. 66, 2665 (1991).

${ }^{14}$ N. Bernhoeft, B. Roessli, N. Sato, N. Aso, A. Hiess, G. H. Lander, Y. Endoh, and T. Komatsubara, Physica B 259-261, 614 (1999).

${ }^{15}$ F. Steglich et al., Physica C 341-348, 691 (2000).

${ }^{16}$ Yu. G. Naidyuk, O. E. Kvitnitskaya, A. G. M. Jansen, P. Wyder, C. Geibel, and A. A. Menovsky, Fiz. Nizk. Temp. 27, 668 (2001) [Low Temp. Phys. 27, 493 (2001)].

${ }^{17}$ N. Sato, Physica B 259-261, 634 (1999).

${ }^{18}$ M. Nicklas, R. Borth, E. Lengyel, P. G. Pagliuso, J. L. Sarrao, V. A. Sidorov, G. Sparn, F. Steglich, and J. D. Thomson, J. Phys. Condens. Matter 13, L905 (2001). 